\title{
Transcriptional response of murine microglia in Alzheimer's disease and inflammation
}

\author{
Daniel C. Shippy ${ }^{1}$, Jyoti J. Watters ${ }^{2}$ and Tyler K. Ulland ${ }^{1 *}$
}

\begin{abstract}
Background: Alzheimer's disease (AD) is a neurodegenerative disorder and is the most common cause of late-onset dementia. Microglia, the primary innate immune cells of the central nervous system (CNS), have a complex role in AD neuropathology. In the initial stages of $A D$, microglia play a role in limiting pathology by removing amyloid- $\beta$ ( $A \beta$ ) by phagocytosis. In contrast, microglia also release pro-inflammatory cytokines and chemokines to promote neuroinflammation and exacerbate AD neuropathology. Therefore, investigating microglial gene networks could identify new targets for therapeutic strategies for AD.

Results: We identified 465 differentially expressed genes (DEG) in 5XFAD versus wild-type mice by microarray, 354 DEG in lipopolysaccharide (LPS)-stimulated N9 microglia versus unstimulated control cells using RNA-sequencing (RNA-seq), with 32 DEG common between both datasets. Analyses of the 32 common DEG uncovered numerous molecular functions and pathways involved in A $\beta$ phagocytosis and neuroinflammation associated with AD. Furthermore, multiplex ELISA confirmed the induction of several cytokines and chemokines in LPS-stimulated microglia.

Conclusions: In summary, AD triggered multiple signaling pathways that regulate numerous genes in microglia, contributing to $A \beta$ phagocytosis and neuroinflammation. Overall, these data identified several regulatory factors and biomarkers in microglia that could be useful in further understanding AD neuropathology.
\end{abstract}

Keywords: Alzheimer's disease, Microglia, RNA-seq, Microarray, Transcriptomics

\section{Background}

Alzheimer's disease (AD) is a neurodegenerative disorder and is the most common cause of late-onset dementia. In the United States, approximately 6.2 million people are living with Alzheimer's dementia, a number estimated to grow to 13.8 million by 2060 unless medical intervention strategies are developed for $\mathrm{AD}[1]$. AD neuropathology is defined by the aggregation of extracellular amyloid- $\beta(A \beta)$ plaques followed by the development of intracellular neurofibrillary tangles (NFTs) composed of hyperphosphorylated tau [2]. In addition to $A \beta$ plaques and tau NFTs, neuroinflammation plays a key role in AD

\footnotetext{
${ }^{*}$ Correspondence: tulland@wisc.edu

${ }^{1}$ Department of Pathology and Laboratory Medicine, University of Wisconsin, Madison, WI, USA

Full list of author information is available at the end of the article
}

neuropathology, promoting numerous inflammatory processes in the central nervous system (CNS) [3].

Microglia, the primary innate immune cells of the $\mathrm{CNS}$, have a complex role in $\mathrm{AD}$ neuropathology. In the early stages of $\mathrm{AD}$, microglia reduce $\mathrm{A} \beta$ accumulation by phagocytosis, and act as a defense barrier to protect plaque adjacent neurons from neurotoxic effects $[4,5]$. Alternatively, microglia can contribute to neuroinflammation by the release of pro-inflammatory cytokines and chemokines, reactive oxygen species, and other molecules associated with increased AD neuropathology [6]. Although the role of microglia in AD is still not entirely understood, it is clear microglia play a key role in the development of AD neuropathology.

Since microglia appear to be an important factor in $\mathrm{AD}$ development, investigating microglial gene networks 
could lead to new therapies to treat AD. Several studies have already highlighted the importance of specific genes involved in microglial metabolism [7] and response to $\mathrm{A} \beta$ plaque pathology [8]. In this study, we investigated the transcriptional response of microglia in an $\mathrm{AD}$ versus non-AD state using microarray and RNA-sequencing (RNA-seq). We identified 465 differentially expressed genes (DEG) in 5XFAD versus wild-type mice, and 354 DEG in lipopolysaccharide (LPS)-stimulated N9 microglia versus unstimulated control cells, with 32 DEG common to both experiments. Of the 32 DEG, functional enrichment analyses identified numerous processes and pathways in which microglia are potentially involved during $\mathrm{AD}$ development. Furthermore, multiplex ELISA confirmed the induction of several cytokines and chemokines in LPS-stimulated microglia that were also differentially expressed in the microarray and RNA-seq datasets. Overall, these data identify novel potential regulatory factors and biomarkers in the microglial response to AD.

\section{Results}

\section{Transcriptome analyses of microglia in AD}

Transcriptional analysis of LPS-stimulated N9 microglia versus non-stimulated control cells was performed using RNA-seq. A total of 354 significant DEG $\left(\log _{2} \mathrm{FC}>1.5\right.$, FDR-adjusted $P$-value $<0.05)$ were identified with 323 upregulated genes and 31 down-regulated genes (Fig. $1 \mathrm{~A}$ and Additional File 1). Interleukin-1 alpha (Il1 $\alpha$ ) was the most up-regulated gene $\left(\log _{2} \mathrm{FC}=9.70\right)$ and albumin $(A l b)$ was the most down-regulated gene $\left(\log _{2} \mathrm{FC}=-7.62\right)$ (Table 1$)$.

We compared this data to publicly available data derived from sorted microglia from female 8-month-old wild-type and 5 XFAD mice, a mouse model of AD which accumulates $A \beta$ plaques [9]. Transcriptional analysis of microglia from 5XFAD versus wild-type mice was performed by microarray [10]. A total of 465 significant gene transcripts $(\mathrm{FC}>2$, FDR-adjusted $P$-value $<0.05)$ were identified with 337 up-regulated gene transcripts and 128 down-regulated gene transcripts (Fig. 1B and Additional File 2). Glycoprotein (transmembrane) nmb (Gpnmb) was

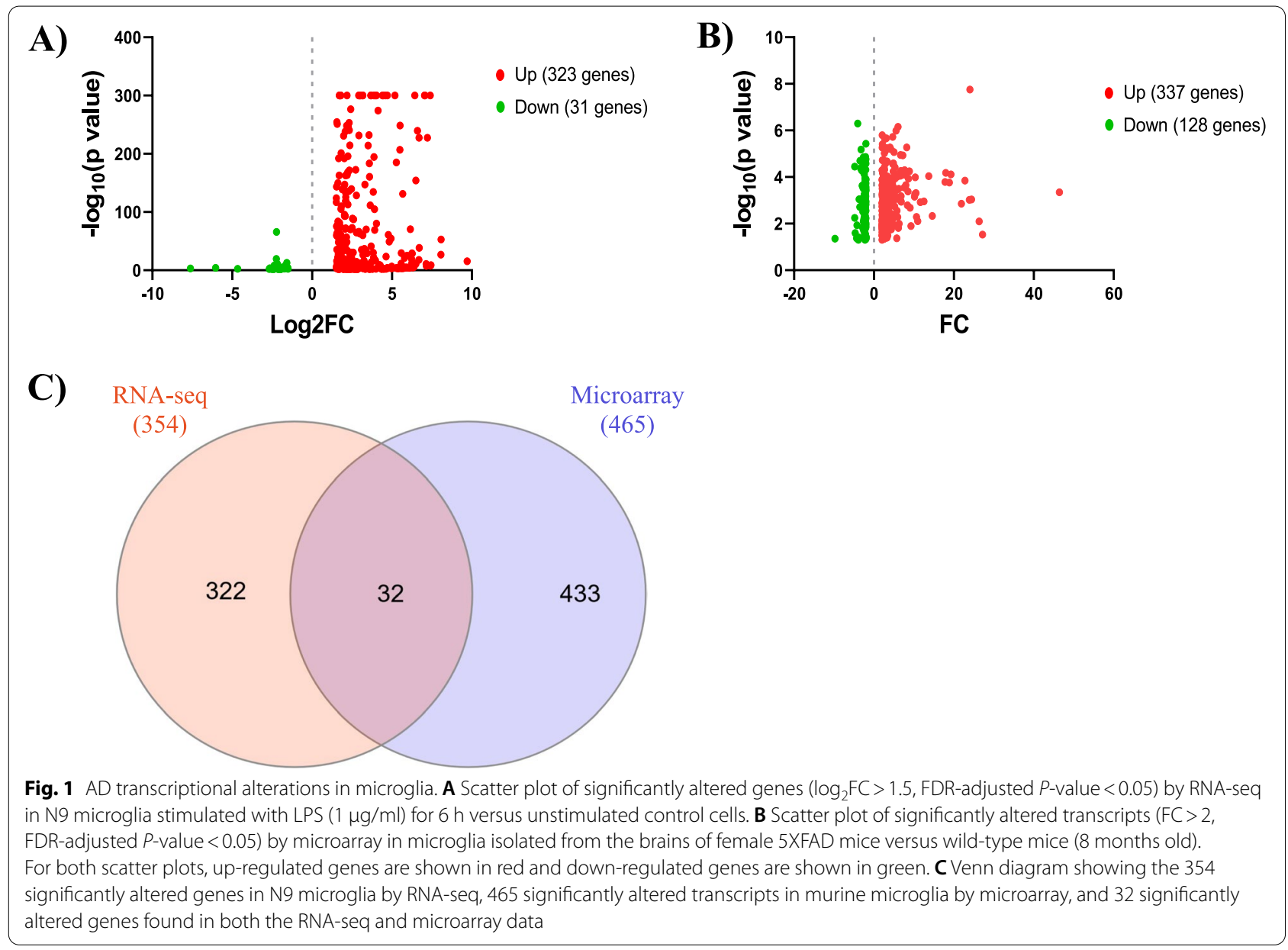


Table 1 Top 10 up-regulated and down-regulated genes in LPS-stimulated N9 microglia versus control cells determined by RNA-seq

\begin{tabular}{|c|c|c|c|}
\hline Gene & Gene ID ${ }^{a}$ & Gene Description & $\log _{2} \mathrm{FC}$ \\
\hline$\| 1 a$ & 27399 & interleukin 1 alpha & 9.70 \\
\hline Ifit3b & 62488 & interferon-induced protein with tetratricopeptide repeats $3 B$ & 8.08 \\
\hline Nos2 & 20826 & nitric oxide synthase 2 , inducible & 8.07 \\
\hline Col5a3 & 04098 & collagen, type V, alpha 3 & 7.44 \\
\hline Rsad2 & 20641 & radical S-adenosyl methionine domain containing 2 & 7.39 \\
\hline Gm19410 & 09372 & predicted gene, 19,410 & 7.39 \\
\hline Ifit3 & 74896 & interferon-induced protein with tetratricopeptide repeats 3 & 7.21 \\
\hline $117 f 9$ & 44103 & interleukin 1 family, member 9 & 7.15 \\
\hline Trim30c & 78616 & tripartite motif-containing $30 \mathrm{C}$ & 7.13 \\
\hline Ifit1 & 34459 & interferon-induced protein with tetratricopeptide repeats 1 & 7.06 \\
\hline Alb & 29368 & albumin & -7.62 \\
\hline Gc & 35540 & vitamin $\mathrm{D}$ binding protein & -6.04 \\
\hline Ttr & 61808 & transthyretin & -4.66 \\
\hline BC021767 & 85006 & cDNA sequence $B C 021767$ & -2.68 \\
\hline T/r8 & 40522 & toll-like receptor 8 & -2.65 \\
\hline 4930473A02Rik & 60029 & RIKEN cDNA 4930473A02 gene & -2.55 \\
\hline Fsbp & 94595 & fibrinogen silencer binding protein & -2.51 \\
\hline Nlrp1a & 69830 & NLR family, pyrin domain containing $1 \mathrm{~A}$ & -2.45 \\
\hline Cgn & 68876 & cingulin & -2.41 \\
\hline$A b c d 2$ & 55782 & ATP-binding cassette, sub-family D (ALD), member 2 & -2.37 \\
\hline
\end{tabular}

all gene IDs start with ENSMUSG000000

Table 2 Top 10 up-regulated and down-regulated gene transcripts in 5XFAD versus wild-type mice determined by microarray

\begin{tabular}{|c|c|c|c|}
\hline Gene & Gene ID ${ }^{a}$ & Gene Description & FC \\
\hline Gpnmb & 031840 & glycoprotein (transmembrane) nmb & 46.43 \\
\hline$D d x 3 y$ & 091190 & DEAD (Asp-Glu-Ala-Asp) box polypeptide 3, Y-linked & 27.16 \\
\hline Spp 1 & 112747 & secreted phosphoprotein 1 & 26.34 \\
\hline Mamdc2 & 036069 & MAM domain containing 2 & 24.41 \\
\hline Cst7 & 089200 & cystatin F (leukocystatin) & 24.03 \\
\hline Fabp3 & 070532 & fatty acid binding protein 3 , muscle and heart & 23.89 \\
\hline Fabp5 & 029046 & fatty acid binding protein 5, epidermal & 22.76 \\
\hline Bhlhe40 & 032194 & basic helix-loop-helix family, member e 40 & 21.89 \\
\hline Hpse & 045617 & heparanase & 19.26 \\
\hline lgf1 & 121161 & insulin-like growth factor 1 & 18.88 \\
\hline XIr4b & 114506 & X-linked lymphocyte-regulated 4B & -9.76 \\
\hline Gpr165 & 033554 & G protein-coupled receptor 165 & -4.84 \\
\hline Snord35b & 082833 & small nucleolar RNA, C/D box 35B & -4.83 \\
\hline Xist & 127786 & inactive $X$ specific transcripts & -4.70 \\
\hline 4933434E20Rik & 159064 & RIKEN cDNA 4933434E20 gene & -4.35 \\
\hline Snord61 & 083176 & small nucleolar RNA, C/D box 61 & -4.25 \\
\hline Bank1 & 041577 & B-cell scaffold protein with ankyrin repeats 1 & -4.08 \\
\hline Ttr & 075312 & transthyretin & -3.90 \\
\hline Fam71a & 171798 & family with sequence similarity 71 , member $A$ & -3.76 \\
\hline $117 r$ & 003981 & interleukin 7 receptor & -3.73 \\
\hline
\end{tabular}

${ }^{a}$ All gene IDs start with ENSMUST00000 
Table 3 Genes found in both the RNA-seq and microarray experiments

\begin{tabular}{|c|c|c|c|c|}
\hline Gene & Gene ID ${ }^{a}$ & Gene Description & RNA-seq $\log _{2} F C$ & Microarray FC \\
\hline Ifit3 & 74896 & interferon-induced protein with tetratricopeptide repeats 3B & 7.21 & 2.21 \\
\hline Cxcl10 & 34855 & chemokine (C-X-C motif) ligand 10 & 7.04 & 3.08 \\
\hline $\mathrm{CxCl} 2$ & 58427 & chemokine (C-X-C motif) ligand 2 & 6.60 & 3.72 \\
\hline $1 / 16$ & 27398 & interleukin 1 beta & 5.94 & 7.76 \\
\hline Oasl2 & 29561 & 2'-5'oligoadenylate synthetase-like 2 & 5.18 & 4.29 \\
\hline Ifit2 & 45932 & interferon-induced protein with tetratricopeptide repeats 2 & 4.58 & 3.15 \\
\hline Ifi204 & 73489 & interferon activated gene 204 & 4.13 & 2.70 \\
\hline Tnf & 24401 & tumor necrosis factor & 3.87 & 2.41 \\
\hline Slfn5 & 54404 & schlafen 5 & 3.67 & 3.57 \\
\hline $\operatorname{Prdm} 1$ & 38151 & PR domain containing 1 , with ZNF domain & 3.29 & 2.57 \\
\hline Slfn2 & 72620 & schlafen 2 & 3.16 & 2.25 \\
\hline $1 / 1 r n$ & 26981 & interleukin 1 receptor antagonist & 2.99 & 4.35 \\
\hline Cd83 & 15396 & CD83 antigen & 2.97 & 2.17 \\
\hline Gvin 1 & 45868 & GTPase, very large interferon inducible 1 & 2.82 & 2.20 \\
\hline Phldal & 20205 & pleckstrin homology like domain, family A, member 1 & 2.75 & 3.85 \\
\hline Gm1966 & 73902 & predicted gene 1966 & 2.44 & 2.19 \\
\hline Gpr84 & 63234 & G protein-coupled receptor 84 & 2.39 & 2.02 \\
\hline Cd69 & 30156 & CD69 antigen & 2.36 & 9.28 \\
\hline Slc7a11 & 27737 & $\begin{array}{l}\text { solute carrier family } 7 \text { (cationic amino acid transporter, y + system), } \\
\text { member } 11\end{array}$ & 2.31 & 2.59 \\
\hline $\mathrm{Cd} 274$ & 16496 & CD274 antigen & 2.09 & 5.72 \\
\hline Irak3 & 20227 & interleukin-1 receptor-associated kinase 3 & 2.06 & 2.20 \\
\hline Serpine1 & 37411 & serine (or cysteine) peptidase inhibitor, clade E, member 1 & 2.03 & 4.16 \\
\hline Mmp12 & 49723 & matrix metallopeptidase 12 & 1.77 & 2.73 \\
\hline T/r2 & 27995 & toll-like receptor 2 & 1.77 & 2.70 \\
\hline Olr1 & 30162 & oxidized low density lipoprotein (lectin-like) receptor 1 & 1.75 & 2.70 \\
\hline Rab11fipl & 31488 & RAB11 family interacting protein 1 (class I) & 1.66 & 2.42 \\
\hline Cd30olf & 47798 & CD300 molecule like family member $F$ & 1.63 & 3.45 \\
\hline C3 & 24164 & complement component 3 & 1.59 & 2.12 \\
\hline $\operatorname{ltg} a 5$ & 00555 & integrin alpha 5 (fibronectin receptor alpha) & 1.57 & 4.85 \\
\hline Bcl2ald & 99974 & B cell leukemia/lymphoma 2 related protein A1d & 1.53 & 4.05 \\
\hline Plaur & 46223 & plasminogen activator, urokinase receptor & 1.51 & 3.60 \\
\hline Ttr & 61808 & transthyretin & -4.66 & -3.9 \\
\hline
\end{tabular}

${ }^{\mathrm{a}}$ All gene IDs start with ENSMUSG000000

the most up-regulated gene transcript $(\mathrm{FC}=46.43)$ and $\mathrm{X}$-linked lymphocyte-regulated 4B (Xlr4b) was the most down-regulated gene transcript $(\mathrm{FC}=-9.76)$ (Table 2).

In total, 32 genes overlapped between the N9 RNA-seq experiment and 5XFAD microarray dataset (Fig. 1C). Of the 32 total genes, 31 were up-regulated and only one was downregulated (Table 3). The majority of the most up-regulated genes were cytokines and chemokines involved in inflammation (Cxcl10, Cxcl2, Il1 $\beta$, Tnf). Transthyretin (Ttr) was the only down-regulated gene common to both datasets.

\section{Pathway and functional prediction of microglia in AD}

Geno ontology (GO), Kyoto Encyclopedia of Genes and Genomes (KEGG), and Search Tool for the
Retrieval of Interacting Genes/Proteins (STRING) analyses were performed on the 32 genes found in both the RNA-seq and microarray datasets. Biological Process (BP) GO indicated the DEG participated in immune system process (Oasl2, Cd30olf, Prdm1, C3, Ifit2, Ifit3, Olr1, Tlr2), immune response (Oasl2, C d274, Cxcl10, Cxcl2, Il1 $\beta$, Tlr2, Tnf), positive regulation of interleukin-8 (Il1 $\beta$, Serpine1, Tlr2, Tnf), response to LPS (Cxcl10, Cxcl2, Il1 $\beta$, Irak3, Tlr2, Thf), inflammatory response $(C x c l 10, C x c l 2, C 3, I l 1 \beta, O l r 1, T l r$ 2, $\operatorname{Tnf})$, positive regulation of gene expression ( $\operatorname{Prdm} 1$, Il1 $\beta$, Plaur, Serpine1, Tlr2, Tnf), innate immune response (Oasl2, Prdm1, C3, Ifit2, Ifit3, Tlr2), regulation of cell proliferation (Prdm1, Cxcl10, Cxcl2, Serpine1, Tnf), positive 
regulation of NF-kappaB transcription factor activity (Il1 $\beta$, Irak3, Tlr2, Tnf), positive regulation of apoptotic process (Bcl2a1d, Ifit2, Il1 $\beta$, Phlda1, Tnf), positive regulation of protein phosphorylation (C3, Il1 $\beta$, Plaur, Tnf), negative regulation of cell proliferation (Ifit3, Il1 $\beta$, Slfn2, Tlr2, Tnf), cellular response to LPS (Cxcl10, Cxcl2, Serpine1, Tnf), negative regulation of gene expression (Prdm1, Il1 $\beta$, Serpine1, Tnf), and signal transduction (Cd274, Cd83, Gpr84, Irak3, Tlr2, Tnf) (Fig. 2A). Cellular Component (CC) GO indicated the DEG were located in the external side of plasma membrane $(C d 274$, Cd69, Cd83, Cxcl10, Itga5, Tlr2, Tnf), extracellular region (Cxcl10, Cxcl2, C3, Il1ß, Il1rn, Mmp12, Olr1, Plaur, Serpine1, Ttr, Tnf), extracellular space (Cxcl10, Cxcl2, C3, Il1ß, Il1rn, Serpine1, Ttr, Tnf), cell surface (Cd274, Itga5, Slc7a11, Tlr2, Tnf), and cytoplasmic vesicle (Rab11fip1, Itga5, Il1 $\beta$, Phlda1, Tlr2) (Fig. 2B). Molecular Function (MF) GO indicated the DEG were involved in cytokine activity (Cxcl10, Cxcl2, Il1 $\beta$, Il1rn, Tnf), CXCR chemokine receptor binding (Cxcl10, $C x c l 2)$, protein binding $(C d 274$, Cd30olf, Prdm1, C3, Itga5, Ifi204, Ifit3, Irak3, Plaur, Serpine1, Tlr2, Ttr, Tnf), interleukin-1 receptor binding (Il1 $\beta$, Illrn), and protein heterodimerization activity (Bcl2ald, Irak3, Tlr2, Ttr) (Fig. 2C).

KEGG analysis was performed on the 32 common genes found in both the microarray and RNA-seq datasets to identify pathways associated with AD. KEGG identified a total of seven pathways $(P<0.05)$ associated with the 32 genes (Fig. 3). The pathways included toll-like receptor signaling (Tlr2, Tnf, Il1 $\beta$, Cxcl10), TNF signaling (Cxcl10, Cxcl2, Il1 $\beta$, Tnf), phagosome (C3, Itga5, Olr1, Tlr2), NOD-like receptor signaling (Cxcl2, Il1 $\beta$, Tnf), cytokine-cytokine receptor interaction (Cxcl10, $C x c l 2, I l 1 \beta, T n f)$, complement and coagulation cascades (C3, Plaur, Serpine1), and NF-kappa B signaling (Bcl2ald, Ill $\beta, T n f)$.

To further understand the interactions of the DEG, we performed protein-protein interaction (PPI) analysis on the 32 genes found in both the RNA-seq and microarray datasets using STRING. For the analysis, text mining, experiments, and databases were chosen for the interaction sources, and the high confidence value of 0.700 was selected as the minimum required interaction score threshold. Of the proteins encoded by the 32 identified DEG, two distinct networks emerged with 15 proteins in one network (IL1 $\beta$, Irak3, Il1rn, Cxcl2, Cxcl10, Tnf, Tlr2, Olr1, Cd83, Cd69, Cd274, Serpine1, Plaur, Itga5, Mmp12), three proteins in the second network (Ifit2, Ifit2, Oasl2), and 13 of the proteins not clustering (Fig. 4). The database did not recognize predicted gene 1966 (Gm1966). The results suggest that the given proteins were highly enriched $\left(P<1 \times 10^{-16}\right)$.

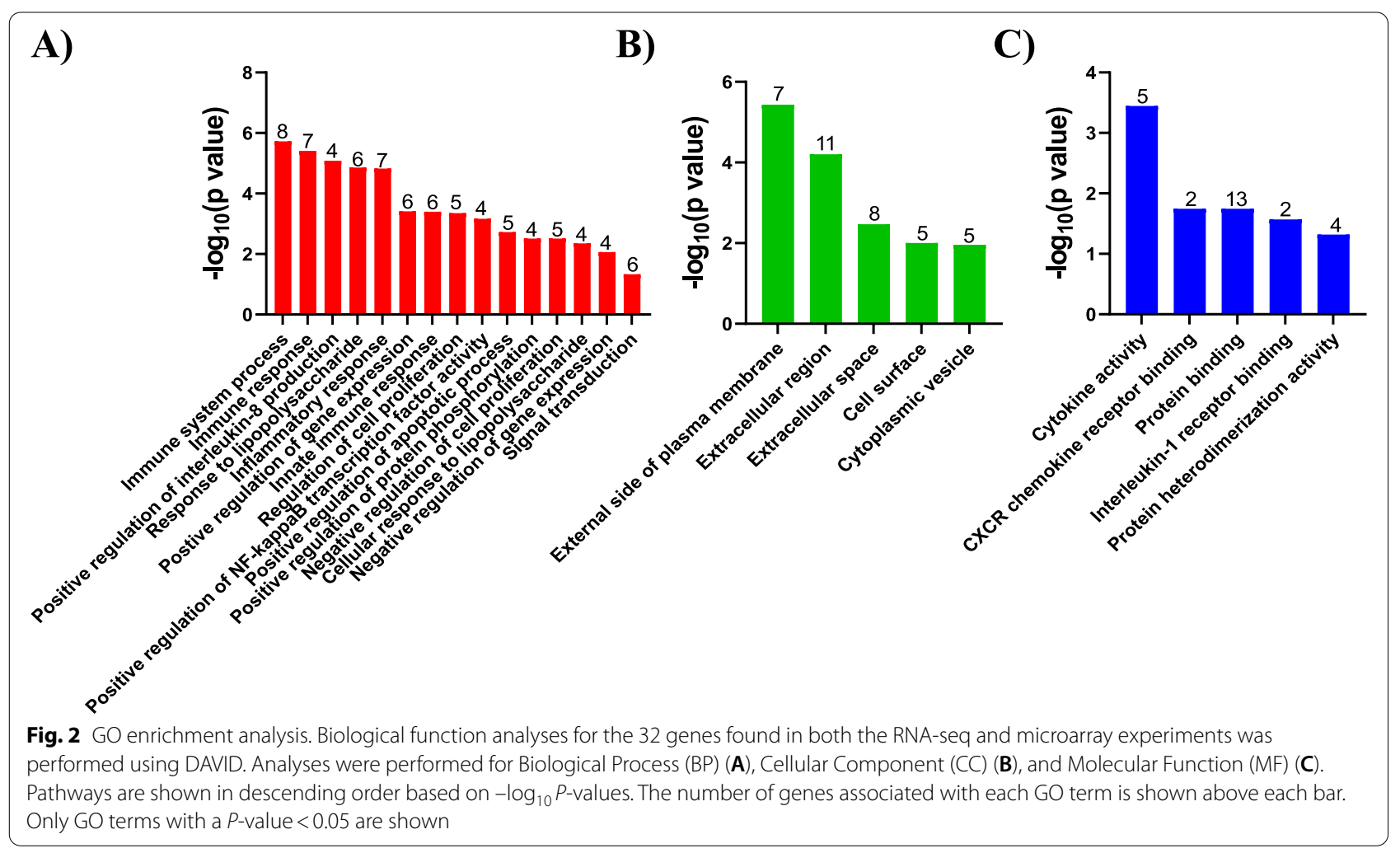




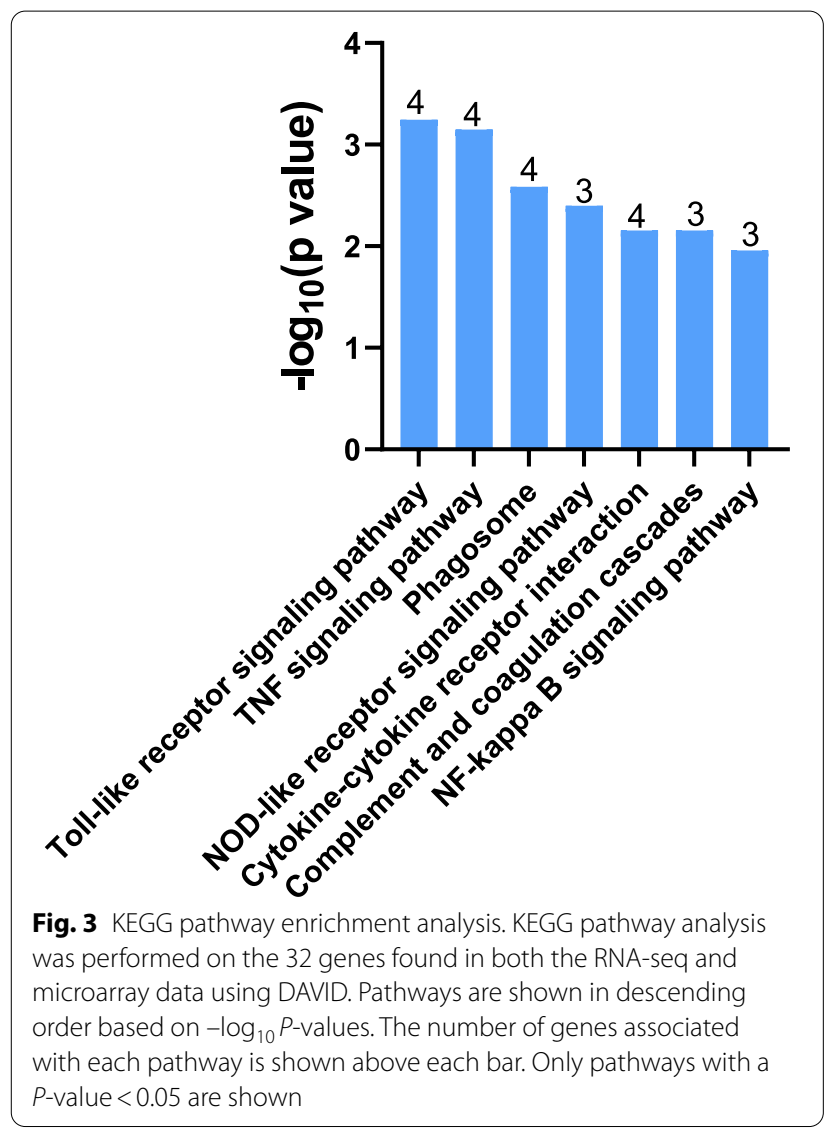

Secretion of cytokines and chemokines by microglia Since so many DEG were cytokines and chemokines associated with inflammation, we performed multiplex ELISAs (22-plex) on supernatants from LPS-stimulated bone marrow-derived macrophages (BMDM), primary microglia, and N9 microglia. In BMDM, IL- $\alpha$, IL- $1 \beta$, IL-2, IL-5, IL-6, IL-10, IL-12, MCP-1, IFN- $\gamma$, TNF- $\alpha$, MIP-1 $\alpha$, GM-CSF, RANTES, KC, MDC, TARC, and TCA-3 showed significant induction when stimulated with LPS versus non-stimulated control cells (Additional File 3). In primary microglia, IL-6, IL-12, TNF- $\alpha$, MIP-1 $\alpha$, GM-CSF, RANTES, KC, and MCP-1 all showed significant induction in LPS-stimulated primary microglia versus non-stimulated control cells (Fig. 5). In N9 microglia, IL-6, MCP-1, TNF- $\alpha$, MIP- $1 \alpha$, and RANTES all showed significant induction in LPS-stimulated N9 microglia versus non-stimulated control cells (Fig. 6). Cytokines and chemokines from BMDM, primary microglia, and N9 microglia that showed no significant difference between LPS-stimulated and control cells are shown in Additional File 4.

\section{Discussion}

A thorough understanding of the molecular characteristics and regulatory pathways of microglia is essential to develop therapeutic strategies for AD. Accordingly, numerous studies have performed transcriptome analyses to elucidate the role of specific genes in microglia important in AD neuropathology, including Trem2 and ApoE [7, 10-13]. Other studies have investigated the transcriptome of microglia under distinct biological activities, such as $A \beta$ plaque phagocytosis [14], tau pathology [15] and metabolism [16]. In this study, we used a two-pronged approach to determine DEG in AD and inflammation. First, we determined the DEG in microglia from 5XFAD mice versus wild-type mice by microarray. We then determined the DEG in LPS-stimulated microglia using RNA-seq. Altogether, 32 DEG overlapped between the two datasets.

Neuroinflammation by microglia is hypothesized to exacerbate AD neuropathology [17]. In our study, several immunological pathways associated with inflammation were identified in $\mathrm{AD}$-associated microglia that correlated with DEG of well-known pro-inflammatory cytokines and chemokines, such as IL-1 $\beta$, TNF, CXCL10, and CXCL2. Caspase-1 dependent IL-1 $\beta$ secretion occurs during NLRP3 inflammasome activation, and activation of the inflammasome has emerged as an important mechanism in chronic neuroinflammation in AD [18]. Furthermore, immunotherapies blocking inflammasome activation are being investigated for use in $\mathrm{AD}$ and other inflammatory diseases [19-21]. BP GO analysis also identified Olr1 as an inflammatory and immune system response gene. Several studies suggest a role for $\mathrm{Olr} 1$ in lipid metabolism, and genetic variation in $O \operatorname{lr} 1$ as a risk factor for AD [22-24], but little is known about $\mathrm{O} / r 1$ in $\mathrm{AD}$-associated neuroinflammation. Furthermore, Olr1 was recognized as a gene involved in the phagosome pathway by KEGG, suggesting an important role in multiple AD pathogenic processes.

Genes not previously characterized in AD were identified by KEGG to be involved in molecular signaling pathways important in AD development. The B cell leukemia/ lymphoma 2 related protein A1d (Bcl2a1d) was identified as being involved in the nuclear factor kappa-light-chainenhancer of activated B cells (NF-kB) signaling pathway. Activation of NF- $\mathrm{B}$ is an important mechanism in chronic neuroinflammation that significantly increases AD pathology [25]. Furthermore, the plasminogen activator, urokinase receptor (Plaur) was recognized as being associated with complement and coagulation cascades. Complement and coagulation cascades are associated with blood-brain barrier dysfunction and AD progression in mice [26] and humans [27]. Characterization of these genes in the context of $\mathrm{AD}$ could further our 


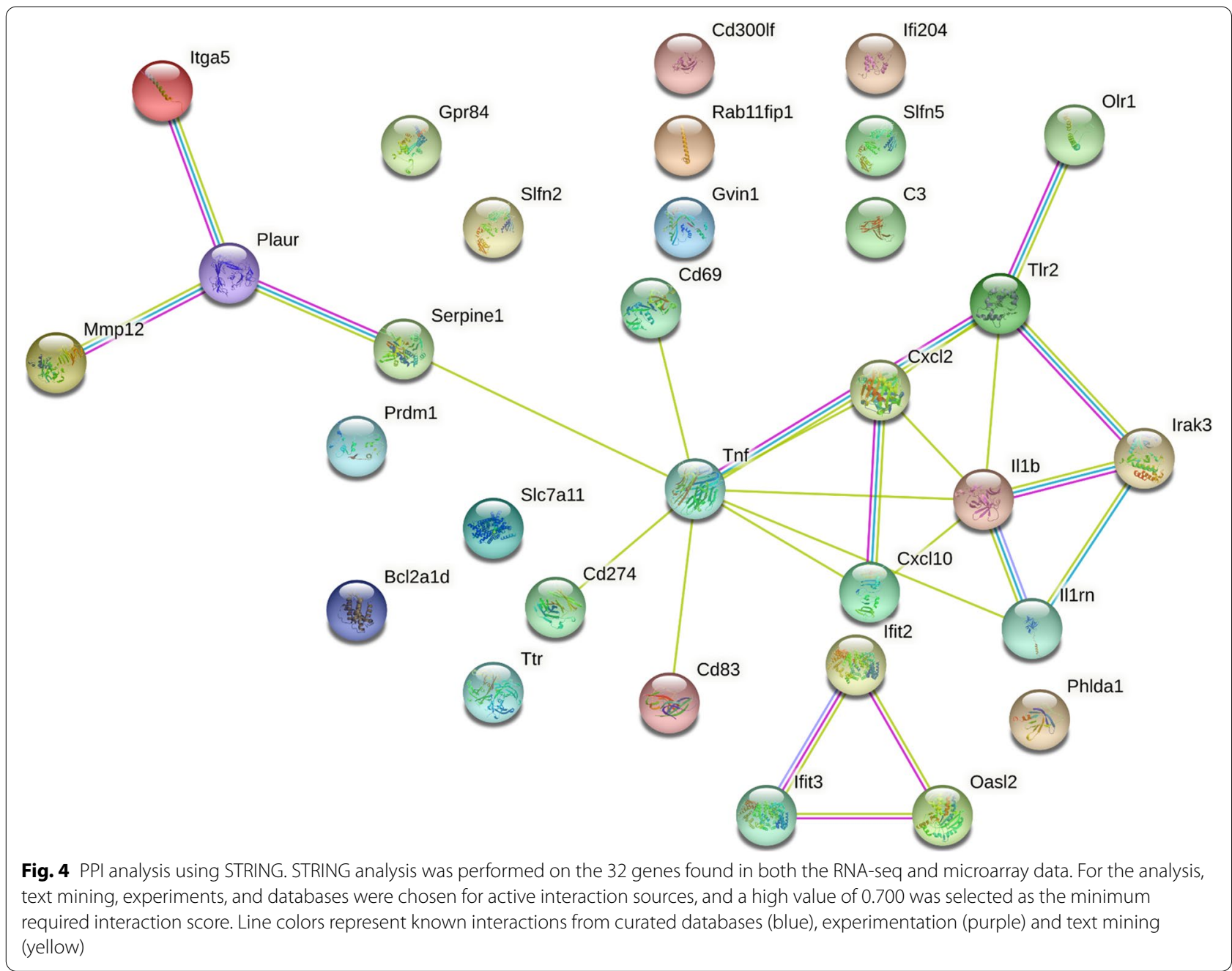

understanding of the underlying regulatory mechanisms in $\mathrm{AD}$ pathogenesis.

Biomarkers can be a useful tool to predict and diagnosis a variety of neurological disorders, including AD [28]. In our study, the only down-regulated gene amongst the 32 DEG was transthyretin (Ttr). Indeed, Ttr has been implicated in numerous processes associated with $\mathrm{AD}$, including $A \beta$ binding [29], $A \beta$ transport at the bloodbrain barrier (BBB) [30], toxicity [31, 32], and neuroprotection by interfering with $A \beta$ formation $[29,33,34]$. Ttr has been previously identified as being decreased in the cerebral spinal fluid (CSF) [35] and blood [36-38] of AD patients, and is being investigated as a blood biomarker for $\mathrm{AD}[38,39]$. Several other DEG in our study could also potentially be used as biomarkers due to their highlevel of differential expression between groups (greater than threefold in both assays), including Ifit3, Cxcl10, Cxcl2, Il1ß, Oasl2, Ifit2, and Slfn5. Further investigation will be needed, as clinically useful biomarkers for $A D$ should be inexpensive, non-invasive, reliably detectable and able to distinguish $\mathrm{AD}$ from other forms of dementia [40].

There are several limitations of our study which are common to most studies directed at identifying dynamic alterations in gene expression networks in AD. Microgliosis is a hallmark of $\mathrm{AD}$ neuropathology, resulting in large numbers of microglia concentrated around $A \beta$ plaques [41]. Since the role of microglia in AD is not fully understood, it is difficult to ascertain whether the altered genes identified in our study are involved in disease progression or in the neuroprotective microglia response. Another caveat to our study is that murine microglia were used in both experiments. Although the value of murine microglial research in AD cannot be overstated, the transcriptional signature of microglia in human $\mathrm{AD}$ is drastically different than that of murine microglia [11, 42]. When comparing human and mouse AD models, transgenic mice overexpress $A \beta$ in a non-physiological manner, resulting in rapid $A \beta$ accumulation and a higher plaque burden when compared to human $\mathrm{AD}[43,44]$. 

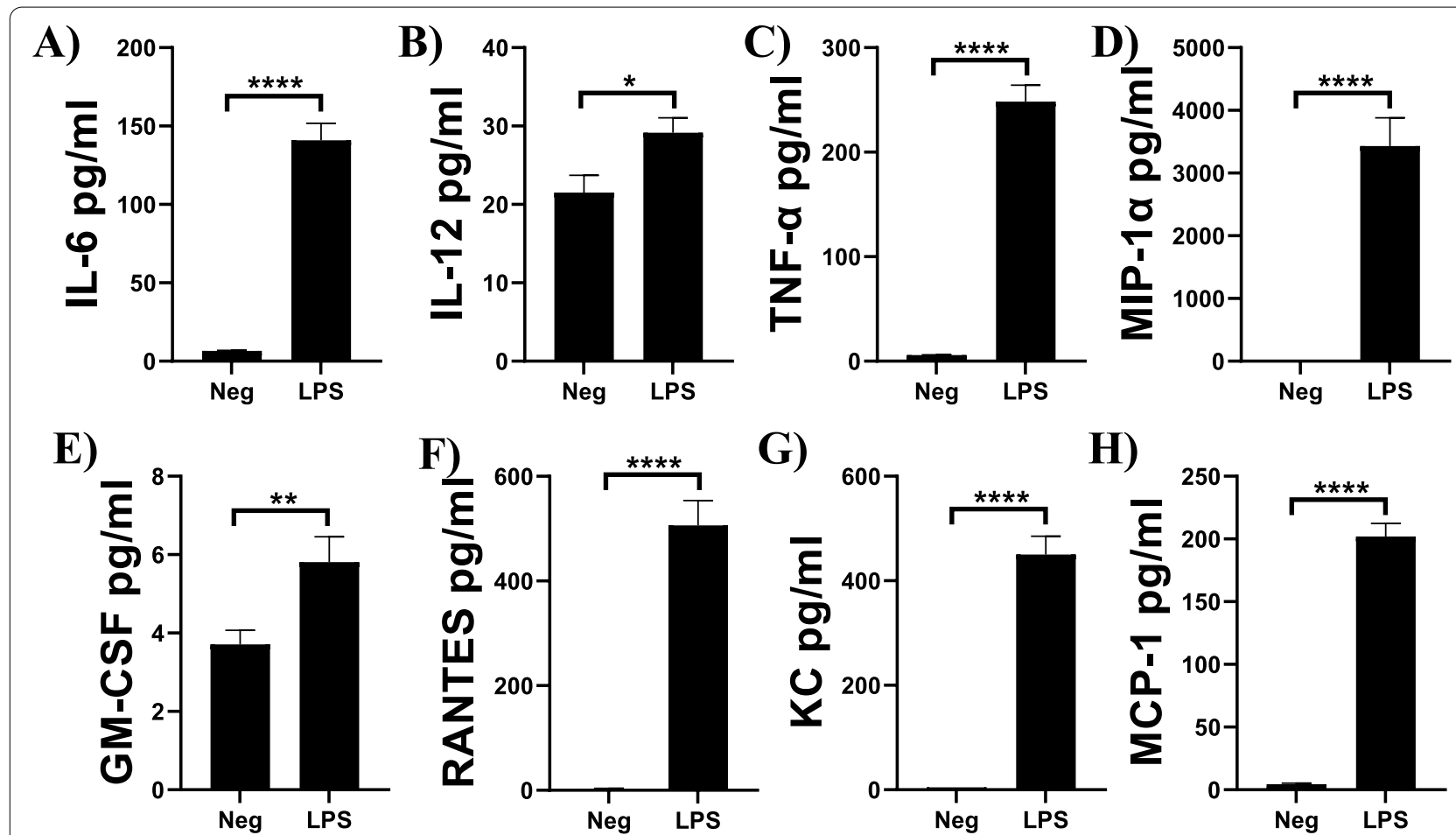

Fig. 5 Cytokines and chemokines induced by LPS in primary microglia. Primary microglia were stimulated with LPS ( $50 \mathrm{ng} / \mathrm{ml})$ for 6 h. Supernatants were collected and assayed for 22 cytokines and chemokines using a multiplex assay. Experiments were performed in triplicate. Results are from three independent experiments. Data are shown as mean \pm SEM. ${ }^{* * *} P \leq 0.0001,{ }^{* *} P \leq 0.01,{ }^{*} P \leq 0.05$

Although the 5XFAD mouse model does not perfectly recapitulate human disease studies, comparing the microglial response in humans and mice have been mixed, with some indicating that the response may be different $[11,42]$ and some studies indicating that the microglial response in both humans and mice may be similar $[7,45]$. This is likely due to differences in the complexity of human disease where both plaques and neurofibrillary tangles are present compared to the 5XFAD mice in which only plaques form. Therefore, in vitro and in vivo models of $\mathrm{AD}$ remain powerful tools to begin to dissect underlying microglial gene regulatory mechanisms in early $\mathrm{AD}$ pathology.

\section{Conclusions}

In summary, we identified 465 DEG in 5XFAD versus wild-type mice by microarray, 354 DEG in LPS-stimulated N9 microglia versus unstimulated control cells using RNA-seq, with 32 DEG common between both data sets. Analyses of the 32 DEG uncovered numerous molecular functions and pathways involved in $A \beta$ phagocytosis and neuroinflammation associated with $\mathrm{AD}$ that may be further investigated. Furthermore, multiplex ELISA confirmed the induction of several cytokines and chemokines in LPS-stimulated microglia. Overall, these data identified several regulatory factors and biomarkers in microglia that could be useful in further understanding $\mathrm{AD}$ neuropathology.

\section{Methods}

\section{Ethical approval and consent to participate}

All methods were carried out in accordance with relevant local and University of Wisconsin guidelines and regulations. All animals were handled in accordance with the Animal Research: Reporting of in vivo Experiments (ARRIVE) guidelines and the University of Wisconsin's Institutional Animal Care and Use Committee policies and our approved protocols.

\section{Cell culture assays}

BMDM were prepared as previously described [19]. Briefly, tibias were removed from wild-type C57BL/6J mice (6-10 months old) (The Jackson Laboratory), flushed with complete RPMI media supplemented with $20 \%$ L-cell conditioned media (LCCM) [19], and cultured for 4-7 days prior to use. Differentiated BMDM were seeded at a cell density of 400,000 cells/well in a 24-well tissue culture plate. Cells were stimulated with ultrapure LPS (50 ng/ml) from Escherichia coli O111:B4 


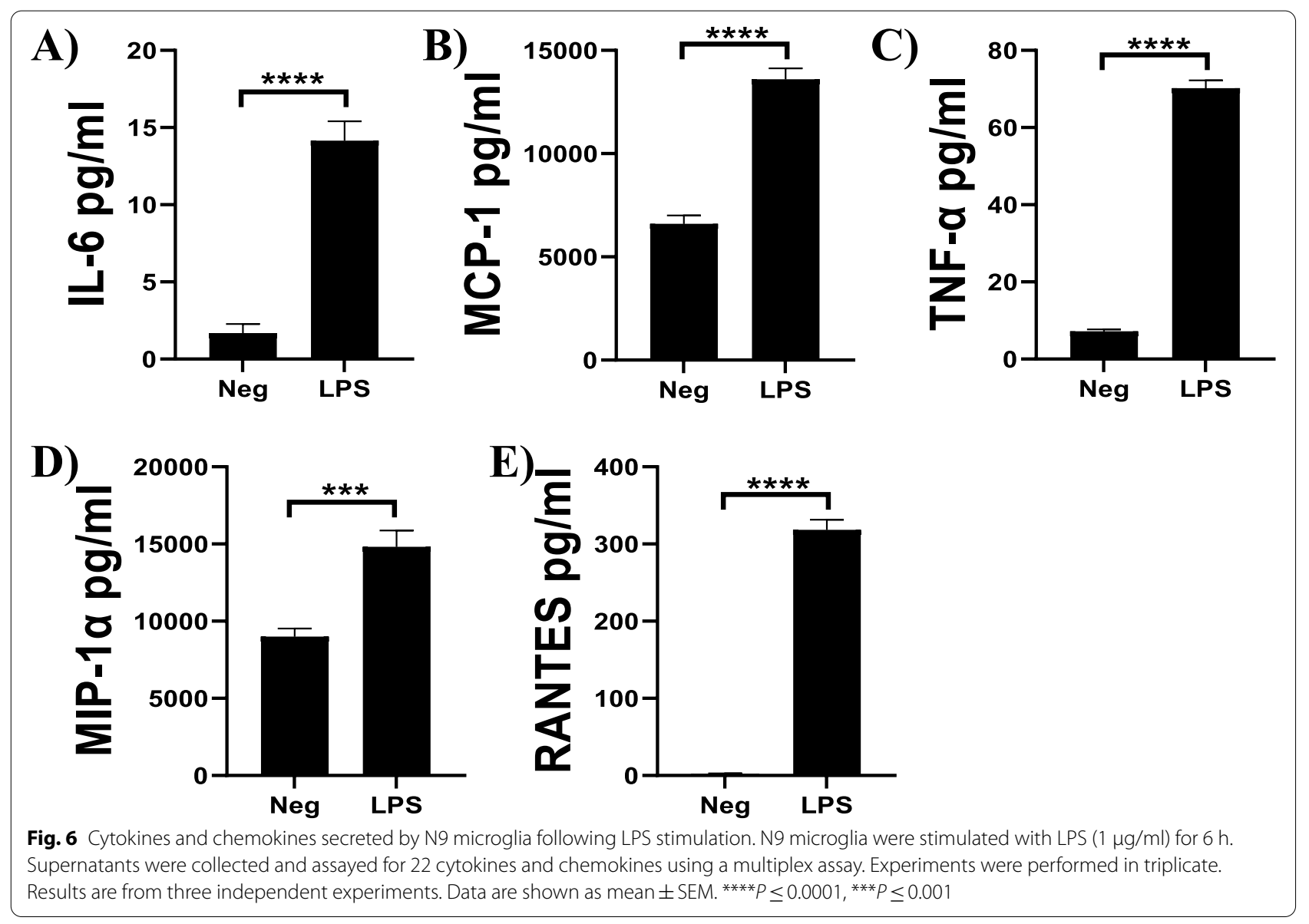

(InvivoGen) for $6 \mathrm{~h}$. Supernatants were flash frozen on dry ice and stored at $-80^{\circ} \mathrm{C}$ until use.

Microglia were prepared from wild-type C57BL/6J mice (5-8 months old) (The Jackson Laboratory) as previously described [7]. Microglia were isolated using Magnetic Activated Cell Sorting (MACS, Miltenyi Biotec) according to manufacturer's instructions. Briefly, mice were perfused with cold PBS containing $0.1 \%$ heparin. Brains were collected in C-tubes (Miltenyi Biotec, Cat. No. 130-096-334) and dissociated using a Neural Tissue Dissociation Kit (T) (Miltenyi Biotec, Cat. No. 130-093-231). Cell suspensions were filtered through a $70 \mu \mathrm{M}$ cell strainer (Corning Falcon ${ }^{\mathrm{TM}}$, Cat. No. 352350). Microglia were labeled with anti-mouse CD45 magnetic beads (Miltenyi Biotec, Cat. No. 130-052-301) and isolated on LS columns (Miltenyi Biotec, Cat. No. 130-042401). LS columns were washed three times with MACS buffer (PBS containing 0.5\% BSA and $1 \mathrm{mM}$ EDTA) before elution. This method allows for preparation of microglial cultures of high purity ( $>95 \%)$. Purity of isolated microglia are routinely confirmed by flow cytometry. Approximately 50,000 microglia/well were seeded on poly-L-lysine coated 24-well plates in complete RPMI media supplemented with 20\% LCCM [19] and human TGF- $\beta$ (10 ng/ml) (PeproTech, Cat. No. 100-21). Media was changed three days after plating, and cells were used in the week following the media change. Microglia were stimulated with LPS $(50 \mathrm{ng} / \mathrm{ml})$ for $6 \mathrm{~h}$. Supernatants were flash frozen on dry ice and stored at $-80^{\circ} \mathrm{C}$ until use.

Immortalized murine N9 microglia were cultured as previously described [46]. N9 microglia were seeded at a cell density of 250,000 cells/well in a 24-well tissue culture plate. Cells were stimulated with LPS $(1 \mu \mathrm{g} / \mathrm{ml})$ [47] for $6 \mathrm{~h}$. Supernatants were flash frozen on dry ice and stored at $-80^{\circ} \mathrm{C}$ until use.

\section{ELISA}

BMDM, primary microglia, and N9 microglial supernatants were assayed for IL- $1 \alpha$, IL-1 $\beta$, IL-2, IL-3, IL-4, IL-5, IL-6, IL-10, IL-12, IL-13, IL-17, MCP-1, IFN- $\gamma$, TNF- $\alpha$, MIP- $1 \alpha$, RANTES, GM-CSF, Eotaxin, KC, MDC, TARC, and TCA-3 using a multiplex assay (Quansys Biosciences). 


\section{RNA-seq}

RNA was extracted from immortalized N9 microglia stimulated with LPS ( $1 \mu \mathrm{g} / \mathrm{ml})$ [47] for $6 \mathrm{~h}$ using a RNeasy Plus Mini Kit (Qiagen, Cat. No. 74134). The N9 microglial cell line is derived from mouse brain and shares numerous phenotypic traits with primary mouse microglia [48]. Quality and quantity of RNA was assessed using an Agilent 2100 Bioanalyzer (Agilent Technologies) and a Nanodrop spectrophotometer (Thermo Scientific). All samples had an RNA integrity number (RIN) of 9.7 or higher. RNA library preparation and transcriptome sequencing were performed by Novogene using the Illumina NovaSeq 6000 Sequencing System. Bioinformatics analysis was performed by Novogene with differential expression analysis performed using the DESeq2 R package (1.20.0). The resulting $P$-values were adjusted using the Benjamini and Hochberg method for controlling the false discovery rate [49]. Genes with FDR-adjusted $P$-values $<0.05$ and $\log 2 \mathrm{FC}>1.5$ were considered differentially expressed.

\section{Microarray}

The microarray has been published in a previous study [10] and the publicly available dataset was used (GSE65067). Briefly, microglia from female 8 month old wild-type $(n=3)$ and 5XFAD $(n=5)$ mice (The Jackson Laboratory) were FACS-sorted directly into RTL-plus lysis buffer. RNA extraction from microglia was performed using a RNeasy Plus Micro Kit (Qiagen, Cat. No. 74034). Microarray hybridization (Affymetrix MoGene 1.0 ST array) and data processing were performed at the Washington University Genome Center. Raw data were normalized using the Robust MultiArray (RMA) method and genes were pre-filtered for expression value greater than or equal to 120 expression units. This method provides a cut-off above which genes have a 95\% chance of expression demonstrated in Immgen dataset, which uses the same array platform [10]. $P$-values were calculated using a Student's t-test and FDR-adjusted $P$-values were calculated using the Benjamini and Hochberg method [49]. Transcripts with FDR-adjusted $P$-values $<0.05$ and FC $>2$ were considered differentially expressed.

\section{Functional enrichment analyses}

Genes found to be differentially expressed in both the RNA-seq and microarray experiments were selected for biological function and pathway analysis. The gene list was uploaded into the Database for Annotation, Visualization and Integrated Discovery (DAVID, v. 6.8) $[50,51]$ for GO and KEGG pathway analysis. GO gene count thresholds of 4,2 , and 2 were used for BP, CC, and MF respectively. Each GO and KEGG pathway with a $P$-value $<0.05$ was considered significant. Additionally, PPI analysis was performed to identify interactions of the selected proteins based on their gene IDs using the STRING database [52]. For the analysis, text mining, experiments, and databases were chosen for active interaction sources using the high confidence (0.700) threshold setting.

\section{Statistical analyses}

Statistical analysis was performed using Prism 9.0.0 (GraphPad). Data are presented as mean \pm SEM. Comparison between two groups was performed using a Student's $t$-test. A $P$-value $\leq 0.05\left({ }^{*} P \leq 0.05, * P \leq 0.01\right.$, *** $P \leq 0.001$ and $\left.{ }^{* * * * P} P \leq 0.0001\right)$ was used as the significance cutoff. The Venn diagram demonstrating overlap in DEG amongst the two datasets was generated using InteractiVenn [53].

\section{Supplementary Information}

The online version contains supplementary material available at https://doi. org/10.1186/s12864-022-08417-8.

\section{Additional file1.}

Additional file2.

Additional file3.

Additional file 4.

\section{Acknowledgements}

We thank Paula Ricciardi-Castagnoli for the N9 microglial cells.

\section{Authors' contributions}

DCS and TKU were responsible for conceptualizing the project, designing, executing, analyzing the results from, and ensuring the validity of the experiments, drafting, and editing the manuscript. JJW supplied reagents, assisted with experimental design, and the drafting and editing of the manuscript. All authors read and approved the final manuscript.

\section{Funding}

This work was funded by NIH R21AG068652 awarded to TKU and R01HL142752-03S1 awarded to TKU and JJW.

\section{Availability of data and materials}

The datasets generated and/or analyzed during the current study are available in the Gene Expression Omnibus (GEO) repository, GSE65067 and GSE183038.

\section{Declarations}

Ethics approval and consent to participate

All methods were carried out in accordance with relevant local and University of Wisconsin guidelines and regulations. All animals were handled in accordance with the Animal Research: Reporting of in vivo Experiments (ARRIVE) guidelines and the University of Wisconsin's Institutional Animal Care and Use Committee policies and our approved protocols. 


\section{Competing interests}

The authors declare that they have no competing interests.

\section{Author details}

1 Department of Pathology and Laboratory Medicine, University of Wisconsin, Madison, WI, USA. ${ }^{2}$ Department of Comparative Biosciences, University of Wisconsin, Madison, WI, USA.

Received: 4 October 2021 Accepted: 21 February 2022

Published online: 05 March 2022

\section{References}

1. 2021 Alzheimer's disease facts and figures. Alzheimers Dement. 2021;17(3):327-406

2. Holtzman DM, Morris JC, Goate AM. Alzheimer's disease: the challenge of the second century. Sci Transl Med. 2011;3(77):77sr71.

3. Leng F, Edison P. Neuroinflammation and microglial activation in Alzheimer disease: where do we go from here? Nat Rev Neurol. 2021;17(3):157-72.

4. Frackowiak J, Wisniewski HM, Wegiel J, Merz GS, Iqbal K, Wang KC. Ultrastructure of the microglia that phagocytose amyloid and the microglia that produce beta-amyloid fibrils. Acta Neuropathol. 1992;84(3):225-33.

5. Condello C, Yuan P, Schain A, Grutzendler J. Microglia constitute a barrier that prevents neurotoxic protofibrillar Abeta42 hotspots around plaques. Nat Commun. 2015;6:6176.

6. Wang WY, Tan MS, Yu JT, Tan L. Role of pro-inflammatory cytokines released from microglia in Alzheimer's disease. Ann Transl Med. 2015;3(10):136.

7. Ulland TK, Song WM, Huang SC, Ulrich JD, Sergushichev A, Beatty WL, Loboda AA, Zhou Y, Cairns NJ, Kambal A, et al. TREM2 Maintains Microglial Metabolic Fitness in Alzheimer's Disease. Cell. 2017;170(4):649-663 e613.

8. Ulrich JD, Ulland TK, Mahan TE, Nystrom S, Nilsson KP, Song WM, Zhou Y, Reinartz M, Choi S, Jiang $H$, et al. ApoE facilitates the microglial response to amyloid plaque pathology. J Exp Med. 2018;215(4):1047-58.

9. Oakley H, Cole SL, Logan S, Maus E, Shao P, Craft J, Guillozet-Bongaarts A, Ohno M, Disterhoft J, Van Eldik L, et al. Intraneuronal beta-amyloid aggregates, neurodegeneration, and neuron loss in transgenic mice with five familial Alzheimer's disease mutations: potential factors in amyloid plaque formation. J Neurosci. 2006;26(40):10129-40.

10. Wang Y, Cella M, Mallinson K, Ulrich JD, Young KL, Robinette ML, Gilfillan S, Krishnan GM, Sudhakar S, Zinselmeyer BH, et al. TREM2 lipid sensing sustains the microglial response in an Alzheimer's disease model. Cell. 2015;160(6):1061-71.

11. Zhou Y, Song WM, Andhey PS, Swain A, Levy T, Miller KR, Poliani PL, Cominelli M, Grover S, Gilfillan S, et al. Human and mouse single-nucleus transcriptomics reveal TREM2-dependent and TREM2-independent cellular responses in Alzheimer's disease. Nat Med. 2020;26(1):131-42.

12. Keren-Shaul H, Spinrad A, Weiner A, Matcovitch-Natan O, Dvir-Szternfeld R, Ulland TK, David E, Baruch K, Lara-Astaiso D, Toth B, et al. A Unique Microglia Type Associated with Restricting Development of Alzheimer's Disease. Cell. 2017;169(7):1276-1290 e1217.

13. Krasemann S, Madore C, Cialic R, Baufeld C, Calcagno N, El Fatimy R, Beckers L, O'Loughlin E, Xu Y, Fanek $Z$, et al. The TREM2-APOE Pathway Drives the Transcriptional Phenotype of Dysfunctional Microglia in Neurodegenerative Diseases. Immunity. 2017;47(3):566-581 e569.

14. Grubman A, Choo XY, Chew G, Ouyang JF, Sun G, Croft NP, Rossello FJ, Simmons R, Buckberry S, Landin DV, et al. Transcriptional signature in microglia associated with Abeta plaque phagocytosis. Nat Commun. 2021;12(1):3015.

15. Wang H, Li Y, Ryder JW, Hole JT, Ebert PJ, Airey DC, Qian HR, Logsdon B, Fisher A, Ahmed Z, et al. Genome-wide RNAseq study of the molecular mechanisms underlying microglia activation in response to pathological tau perturbation in the rTg4510 tau transgenic animal model. Mol Neurodegener. 2018;13(1):65.

16. Baik SH, Kang S, Lee W, Choi H, Chung S, Kim Jl, Mook-Jung I. A Breakdown in Metabolic Reprogramming Causes Microglia Dysfunction in Alzheimer's Disease. Cell Metab. 2019;30(3):493-507 e496.

17. Heneka MT, Carson MJ, El Khoury J, Landreth GE, Brosseron F, Feinstein DL, Jacobs AH, Wyss-Coray T, Vitorica J, Ransohoff RM, et al. Neuroinflammation in Alzheimer's disease Lancet Neurol. 2015;14(4):388-405.

18. Heneka MT, Kummer MP, Stutz A, Delekate A, Schwartz S, Vieira-Saecker A, Griep A, Axt D, Remus A, Tzeng TC, et al. NLRP3 is activated in Alzheimer's disease and contributes to pathology in APP/PS1 mice. Nature. 2013;493(7434):674-8.

19. Shippy DC, Wilhelm C, Viharkumar PA, Raife TJ, Ulland TK. beta-Hydroxybutyrate inhibits inflammasome activation to attenuate Alzheimer's disease pathology. J Neuroinflammation. 2020;17(1):280.

20. Goldberg EL, Asher JL, Molony RD, Shaw AC, Zeiss CJ, Wang C, MorozovaRoche LA, Herzog RI, Iwasaki A, Dixit VD. beta-Hydroxybutyrate Deactivates Neutrophil NLRP3 Inflammasome to Relieve Gout Flares. Cell Rep. 2017;18(9):2077-87.

21. Youm YH, Nguyen KY, Grant RW, Goldberg EL, Bodogai M, Kim D, D’Agostino D, Planavsky N, Lupfer C, Kanneganti TD, et al. The ketone metabolite beta-hydroxybutyrate blocks NLRP3 inflammasome-mediated inflammatory disease. Nat Med. 2015;21(3):263-9.

22. Lambert JC, Luedecking-Zimmer E, Merrot $S$, Hayes A, Thaker U, Desai $P$, Houzet A, Hermant X, Cottel D, Pritchard A, et al. Association of 3'-UTR polymorphisms of the oxidised LDL receptor 1 (OLR1) gene with Alzheimer's disease. J Med Genet. 2003;40(6):424-30.

23. Wang ZT, Zhong XL, Tan MS, Wang HF, Tan CC, Zhang W, Zheng ZJ, Kong $L L$, Tan L, Sun L. Association of lectin-like oxidized low density lipoprotein receptor 1 (OLR1) polymorphisms with late-onset Alzheimer disease in Han Chinese. Ann Transl Med. 2018;6(10):172.

24. Luedecking-Zimmer E, DeKosky ST, Chen Q, Barmada MM, Kamboh MI. Investigation of oxidized LDL-receptor 1 (OLR1) as the candidate gene for Alzheimer's disease on chromosome 12. Hum Genet. 2002;111(4-5):443-51.

25. Granic I, Dolga AM, Nijholt IM, van Dijk G, Eisel UL. Inflammation and NF-kappaB in Alzheimer's disease and diabetes. J Alzheimers Dis. 2009;16(4):809-21.

26. Ujiie M, Dickstein DL, Carlow DA, Jefferies WA. Blood-brain barrier permeability precedes senile plaque formation in an Alzheimer disease model. Microcirculation. 2003;10(6):463-70.

27. Ryu JK, McLarnon JG. A leaky blood-brain barrier, fibrinogen infiltration and microglial reactivity in inflamed Alzheimer's disease brain. J Cell Mol Med. 2009;13(9A):2911-25.

28. Mayeux R. Biomarkers: potential uses and limitations. NeuroRx. 2004;1(2):182-8.

29. Schwarzman AL, Gregori L, Vitek MP, Lyubski S, Strittmatter WJ, Enghilde JJ, Bhasin R, Silverman J, Weisgraber KH, Coyle PK, et al. Transthyretin sequesters amyloid beta protein and prevents amyloid formation. Proc Natl Acad Sci U S A. 1994;91(18):8368-72.

30. Alemi M, Gaiteiro C, Ribeiro CA, Santos LM, Gomes JR, Oliveira SM, Couraud PO, Weksler B, Romero I, Saraiva MJ, et al. Transthyretin participates in beta-amyloid transport from the brain to the liver-involvement of the low-density lipoprotein receptor-related protein 1? Sci Rep. 2016;6:20164

31. Cascella R, Conti S, Mannini B, Li X, Buxbaum JN, Tiribilli B, Chiti $F$, Cecchi $C$. Transthyretin suppresses the toxicity of oligomers formed by misfolded proteins in vitro. Biochim Biophys Acta. 2013:1832(12):2302-14.

32. Costa R, Goncalves A, Saraiva MJ, Cardoso I. Transthyretin binding to A-Beta peptide-impact on A-Beta fibrillogenesis and toxicity. FEBS Lett. 2008;582(6):936-42

33. Li X, Zhang X, Ladiwala AR, Du D, Yadav JK, Tessier PM, Wright PE, Kelly JW, Buxbaum JN. Mechanisms of transthyretin inhibition of beta-amyloid aggregation in vitro. J Neurosci. 2013;33(50):19423-33.

34. Nilsson L, Pamren A, Islam T, Brannstrom K, Golchin SA, Pettersson N, lakovleva I, Sandblad L, Gharibyan AL, Olofsson A. Transthyretin Interferes with Abeta Amyloid Formation by Redirecting Oligomeric Nuclei into Non-Amyloid Aggregates. J Mol Biol. 2018;430(17):2722-33.

35. Serot JM, Christmann D, Dubost T, Couturier M. Cerebrospinal fluid transthyretin: aging and late onset Alzheimer's disease. J Neurol Neurosurg Psychiatry. 1997:63(4):506-8.

36. Ribeiro CA, Santana I, Oliveira C, Baldeiras I, Moreira J, Saraiva MJ, Cardoso I. Transthyretin decrease in plasma of $\mathrm{MCl}$ and $\mathrm{AD}$ patients: investigation of mechanisms for disease modulation. Curr Alzheimer Res. 2012;9(8):881-9. 
37. Han SH, Jung ES, Sohn JH, Hong HJ, Hong HS, Kim JW, Na DL, Kim M, Kim $\mathrm{H}, \mathrm{Ha} \mathrm{HJ}$, et al. Human serum transthyretin levels correlate inversely with Alzheimer's disease. J Alzheimers Dis. 2011;25(1):77-84.

38. Velayudhan L, Killick R, Hye A, Kinsey A, Guntert A, Lynham S, Ward M, Leung R, Lourdusamy A, To AW, et al. Plasma transthyretin as a candidate marker for Alzheimer's disease. J Alzheimers Dis. 2012;28(2):369-75.

39. Bradley-Whitman MA, Abner E, Lynn BC, Lovell MA. A Novel Plasma Based Biomarker of Alzheimer's Disease. J Alzheimers Dis. 2015;47(3):761-71.

40. Consensus report of the Working Group on: "Molecular and Biochemical Markers of Alzheimer's Disease". The Ronald and Nancy Reagan Research Institute of the Alzheimer's Association and the National Institute on Aging Working Group. Neurobiol Aging. 1998;19(2):109-16.

41. Sarlus $\mathrm{H}$, Heneka MT. Microglia in Alzheimer's disease. J Clin Invest. 2017;127(9):3240-9.

42. Olah M, Menon V, Habib N, Taga MF, Ma Y, Yung CJ, Cimpean M, Khairallah A, Coronas-Samano G, Sankowski R, et al. Single cell RNA sequencing of human microglia uncovers a subset associated with Alzheimer's disease. Nat Commun. 2020;11(1):6129.

43. Drummond E, Wisniewski T. Alzheimer's disease: experimental models and reality. Acta Neuropathol. 2017;133(2):155-75.

44. Liu P, Reichl JH, Rao ER, McNellis BM, Huang ES, Hemmy LS, Forster CL, Kuskowski MA, Borchelt DR, Vassar R, et al. Quantitative Comparison of Dense-Core Amyloid Plaque Accumulation in Amyloid-beta Protein Precursor Transgenic Mice. J Alzheimers Dis. 2017;56(2):743-61.

45. Sobue A, Komine O, Hara Y, Endo F, Mizoguchi H, Watanabe S, Murayama S, Saito T, Saido TC, Sahara N, et al. Microglial gene signature reveals loss of homeostatic microglia associated with neurodegeneration of Alzheimer's disease. Acta Neuropathol Commun. 2021;9(1):1.

46. Righi M, Mori L, De Libero G, Sironi M, Biondi A, Mantovani A, Donini SD, Ricciardi-Castagnoli P. Monokine production by microglial cell clones. Eur J Immunol. 1989;19(8):1443-8.

47. Nikodemova M, Watters JJ. Outbred ICR/CD1 mice display more severe neuroinflammation mediated by microglial TLR4/CD14 activation than inbred C57BI/6 mice. Neuroscience. 2011;190:67-74.

48. Stansley B, Post J, Hensley K. A comparative review of cell culture systems for the study of microglial biology in Alzheimer's disease. J Neuroinflammation. 2012;9:115.

49. Benjamini Y, Hochberg Y. Controlling the False Discovery Rate - a Practical and Powerful Approach to Multiple Testing. J R Stat Soc B. 1995;57(1):289-300.

50. da Huang W, Sherman BT, Lempicki RA. Systematic and integrative analysis of large gene lists using DAVID bioinformatics resources. Nat Protoc. 2009;4(1):44-57.

51. da Huang W, Sherman BT, Lempicki RA. Bioinformatics enrichment tools: paths toward the comprehensive functional analysis of large gene lists. Nucleic Acids Res. 2009;37(1):1-13.

52. Szklarczyk D, Gable AL, Lyon D, Junge A, Wyder S, Huerta-Cepas J, Simonovic M, Doncheva NT, Morris JH, Bork P, et al. STRING v11: protein-protein association networks with increased coverage, supporting functional discovery in genome-wide experimental datasets. Nucleic Acids Res. 2019;47(D1):D607-13.

53. Heberle H, Meirelles GV, da Silva FR, Telles GP, Minghim R. InteractiVenn: a web-based tool for the analysis of sets through Venn diagrams. BMC Bioinformatics. 2015;16:169.

\section{Publisher's Note}

Springer Nature remains neutral with regard to jurisdictional claims in published maps and institutional affiliations.

Ready to submit your research? Choose BMC and benefit from:

- fast, convenient online submission

- thorough peer review by experienced researchers in your field

- rapid publication on acceptance

- support for research data, including large and complex data types

- gold Open Access which fosters wider collaboration and increased citations

- maximum visibility for your research: over $100 \mathrm{M}$ website views per year

At BMC, research is always in progress.

Learn more biomedcentral.com/submissions 